\title{
Development of Capsules Filled with Phenytoin and Berberine Loaded Nanoparticles- A New Approach to Improve Anticonvulsant Efficacy
}

\author{
Chitra Karthikeyini Senthilvel, Kavitha Karuppaiyan*, Muthu Mohammed Jamal Moideen \\ Department of Pharmaceutical Technology, BIT campus, Anna University, Tiruchirappalli, Tamil Nadu, INDIA.
}

\begin{abstract}
Introduction: Currently used anticonvulsant drugs are not totally effective to control seizures. Phenytoin is a classic instance where its efficiency is inhibited through high metabolization (90\%-95\%) and back transport through cytochrome P450 and P-glycoprotein, respectively. To explore and attain improved anticonvulsant efficacy combination therapy with nanotechnology has been adopted in this research. Objective: development of nanotechnology-based drug delivery system by filling berberine and phenytoin nanoparticles in capsules. Method: phenytoin and berberine nanoparticles were formulated individually by utilising a solvent evaporation technique and later the mixture is filled in the hard gelatine capsule to create a single-unit dosage. Results: The formulated nanoparticles were evaluated in terms of FTIR and DSC studies, mean particle diameter and zeta-potential, entrapment efficiency and in vitro release. FTIR and DSC studies had proved that formulation components are compatible. SEM report revealed that all the nanoparticles were smooth, spherical in shape and within the size range of $100-500 \mathrm{~nm}$. Zeta-potential of phenytoin and berberine nanoparticles was negative $(-2.61$ and $-35.9 \mathrm{mV}$, respectively). In vitro release kinetics was in the accordance with the Higuchi model. The improved efficacy was proved by MES and Histopathological studies. Conclusion: The successfully developed Capsules filled with the Nanoparticles (Combination therapy) exhibited improved efficacy than that of single phenytoin therapy.
\end{abstract}

Key words: Status epilepticus (SE), Phenytoin, Berberine, Nanoparticles, Cytochrome P-450, P-Gp efflux transporters.

\section{INTRODUCTION}

Status epilepticus is a serious neurological disorder that results from abnormal/excessive or synchronous neuronal activity of the brain. Normally a raised concentration of calcium ion in neuronal tissue triggers a cascade of biochemical responses that leads to the death of neuronal cell following status epilepticus ${ }^{1}$ and subsequently leads to cellular damage, disruptions and eventually to cell death. ${ }^{2}$ The mortality and morbidity rate of the status epileptics is higher due to the epileptic focal point of cerebral neurons that frequently fires and thus had raised metabolic demands. It has been found that global occurrence of active epilepsy has extended to $4-5$ per $100^{3}$ whereas
WHOs report stated that, around 50 million people are affected. ${ }^{4}$ Since termination of seizure activities and control of brain damage are quite difficult to attain, prompt and safer efficacious therapy is needed to prevent loss of cerebral auto regulation and neuronal damage.

More than $20-40 \%$ epileptic patients are being restricted to anti-epileptic drugs. ${ }^{5}$ The significant issue in the most anticonvulsant drugs has been identified as the efficacy. Phenytoin is the most widely used drug in tonic clonic seizure and status epileptics ${ }^{6,7}$ This drug is a hydantoin derivative under barbiturates group and its efficacy is less due to the metabolisation of more than $90 \%$ of
Submission Date: 07-10-2018; Revision Date: 28-12-2018; Accepted Date: 21-03-2019

DOI: 10.5530/ijper.53.3.79 Correspondence: Dr. Kavitha Karuppaiyan, Department of Pharmaceutical technology, Anna University, Thiruchirappalli620024, Tamil Nadu, INDIA Phone: +919841954918 E-mail:kavithaaut@gmail. com

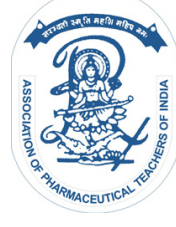

www.ijper.org 
the drug into inactive 5-(p-hydroxyphenyl)-5-phenylhydantoinby cytochrome $\mathrm{P} 450^{8}$ and back transportation by P-glycoprotein (P-gP) efflux transporter.'

The aim of this research is to explore and attain improved anticonvulsant efficiency of combination therapy through the development of nanotechnologybased drug delivery system incorporating berberine and phenytoin. Berberineis anisoquinoline alkaloid, natural COX-2 inhibitor, ${ }^{10}$ P-Gp inhibitor, ${ }^{11}$ cytochrome P450 inhibitor and potent inhibitor of CYP1A and CYP3 $\mathrm{A}^{12,13}$ and therefore it has the potentialto maximize the free drug concentration by reducing the metabolization and back transportation of phenytoin. It also exerts other activities such as anti-inflammatory, anti-diabetic, lipid per-oxidation, neuro-protective activity and most importantly anticonvulsant activity. ${ }^{14,15}$ Subsequently the next strategy was 'adaptation of nanotechnology which positively impacts the efficacy improvement since nanotechnology is a powerful platform and most promising strategy to CNS drug delivery, by which the drugs are efficiently delivered to the brain, by crossing the blood brain barrier. ${ }^{16}$

By utilising both the strategies, the phenytoin and berberine nanoparticles were formulated (Individually) through solvent evaporation and sonication methods and they were loaded in the 1" sized hard gelatine capsules designed as a single unit dosage and later on the nanoparticles were optimized by in vitro release and entrapment efficiency. Since, phenytoin has low solubility, high permeability and of a class II category as per BCS classification ${ }^{17}$ the PEG $6000^{18}$ (solubility enhancer) and HPMC E1 $5^{19}$ carrier were used to formulate phenytoin nanoparticles. Similarly, berberine has low solubility, low permeability and of class IV category and hence to enhance the permeability and solubility, sodium caprate ${ }^{20}$ and PEG 6000 were used respectively. Likewise, HPMC E15 was used as an effective controllrelease carrier which has excellent biocompatibility and biodegradability to provide hydrophilic environment in drugs with intermolecular hydrogen bonding and semi inter penetrating network in the formulation.

\section{MATERIALS AND METHODS}

\section{Methodology}

\section{Research Design}

Since the current research is based on analysing and exploring the effects of drugs in laboratory experimental research design has been adapted by the researcher.

\section{Research Approach}

The research approach adapted by the researcher would be quantitative approach.

\section{Sampling}

- The components Phenytoin and Hydroxyl Propyl Methyl Cellulose $\mathrm{E}_{15}$ were obtained from Medopharm, Chennai (India), at free of cost.

- Sodium Caprate and berberine chloride were purchased from Sigma Chemical Co., USA.

- PEG 6000 was procured as a sample-gift from Matrix, India.

- Methanol and Ethanol were purchased from Merck (India).

- Other reagents (Analytical grade) were purchased from SD Fine chem. (Bangalore).

The obtained samples were subjected to several studies, such as: SEM study, DSC Study and histopathological study, towards attaining the research objectives.

\section{Pre-Formulation Study}

The pre-formulation study in this research makes use of groups of studies like solubility study in which the enhancers' solubility would be studied at equilibrium; similarly, the permeability test was conducted to check the permeability enhancer's concentration optimization; etc.

\section{Solubility Study}

Three solubility enhancers were taken as variables for the test that has been adapted from the study of Higuchi and Connors. ${ }^{21}$ With an excess of drug (200mg), Phenytoin was added to $10 \mathrm{~mL}$ of distilled water that has different concentration of solubility enhancers: PEG 6000, Tween 80 and SLS (0.5\%, 1.0\%, 1.5\% and 2.0\%). The shake-flask method was utilised for separation of phases, for $24 \mathrm{~h}$. When equilibrium was achieved, supernatant liquid was removed and filtered through $0.22 \mu \mathrm{m}$ filter paper, which was later analyzed spectrophotometrically at $205 \mathrm{~nm}$, by Shimadzu UV -1800 visible spectrophotometer. The same solubility test was repeated thrice and the same techniques were carried out for berberine except it was spectrophotometrically analysed at $345 \mathrm{~nm}$.

\section{Permeability Study}

\section{Egg membrane preparation}

The permeability study was carried out to optimize the concentration of permeability enhancer in the formulation. Egg membrane was prepared by soaking the egg in $0.1 \mathrm{~N} \mathrm{HCl}$ overnight after which the egg membrane was peeled off and utilized for permeability test. ${ }^{21}$

This experiment was carried out in Franz diffusion cells at $37^{\circ} \mathrm{C}$. The egg membrane subsequently increased among donor as well as receptor cells after it has been equilibrated with phosphate buffer 6.8. Donor cell berberine and sodium caprate in different ratios $(1: 0,1: 0.2$, 
1:0.4, 1:0.8 and 1:1) were taken separately with 6.8 phosphate buffer $2 \mathrm{~mL}$ and the receptor sections were filled by phosphate buffer and mixed with a layered magnetic bar. Samples were extracted from receptor sections at pre-determined time intervals and then replaced by the same quantity of phosphate buffer. The amount of permeated drug calculated according to the drug concentration in receptor medium determined spectrophotometrically at 345nm (Shimadzu UV-1800 visible Spectrophotometer).

\section{Preparation of Nanoparticles}

\section{Preparation of Phenytoin-Loaded Nanoparticles}

Phenytoin nanoparticles were prepared by solvent evaporation method. ${ }^{22}$ Required HPMC $\mathrm{E}_{15}$ was weighed and dissolved in $10 \mathrm{ml}$ of distilled water and later 1.25 $\%$ PEG 6000 was added with the mixture. Likewise, weighed phenytoin drug was dissolved in $5 \mathrm{~mL}$ of ethanol and was injected into aqueous HPMC E15 solution in mechanical stirrer till it was mixed uniformly. The mixture was later on poured and entirely dried up in a flask of rotary evaporator on a rotating speed with 80 $\mathrm{rpm}$ with the water bath at $60^{\circ} \mathrm{C}$. The obtained dry film on the walls of the rotating flask was shaken with $5 \mathrm{~mL}$ of ethanol and the resulting suspension was subjected to sonication at $60 \%$ amplitude for $60 \mathrm{sec}$ in a probe sonicator. Then, the sediment is removed by centrifugation at $10,000 \mathrm{rpm}$ for $15 \mathrm{~min}$ at $37^{\circ} \mathrm{C}$ and finally the sedimented phenytoin nanoparticles were dried. Formulation parameters are reported in the Table 1.

\section{Preparation of Berberine-Loaded Nanoparticles}

Berberine-loaded nanoparticles were also prepared by the same solvent evaporation method as phenytoinloaded nanoparticles. Accurately weighed quantities of berberine were liquefied in ethanol and the solution was mixed with the aqueous solution of HPMC E15, PEG6000 and sodium caprate and magnetically stirred until the mixture was uniform. The resultant mixture was then placed in rotary evaporator at a speed of $80 \mathrm{rpm}$ at $60^{\circ} \mathrm{C}$; which was later subjected to probe sonication for $60 \mathrm{sec}$ and centrifugation at $10000 \mathrm{rpm}$ for $15 \mathrm{~min}$. finally the resultant pellet that contains berberine-loaded nanoparticles were dried. Formulation parameters are reported in the Table 2.

\section{Nanoparticle characterisation}

\section{UV Spectroscopy (Determination of $\lambda$ max)}

Stock solution of phenytoin and berberine were prepared with methanol. Dilutions were made with methanol at the concentration of $10 \mathrm{mcg} / \mathrm{ml}$; where the UV spectrum was recorded in the range of $200-400 \mathrm{~nm}$ by using Shimadzu spectrophotometer.
Table 1: Preparation of Phenytoin Nanoparticles.

\begin{tabular}{|c|c|c|}
\hline FORMULATION CODE & $\begin{array}{c}\text { DRUG: HPMC } \\
\text { E15 }\end{array}$ & PEG6000 \\
\hline PNANO1 & $1: 0.25$ & 1.25 \\
\hline PNANO2 & $1: 0.5$ & 1.25 \\
\hline PNANO3 & $1: 0.75$ & 1.25 \\
\hline PNANO4 & $1: 1.0$ & 1.25 \\
\hline PNANO5 & $1: 1.25$ & 1.25 \\
\hline PNANO6 & $1: 1.5$ & 1.25 \\
\hline
\end{tabular}

Table 2: Preparation of Berberine Nanoparticles.

\begin{tabular}{|c|c|c|c|}
\hline $\begin{array}{c}\text { FORMULATION } \\
\text { CODE }\end{array}$ & $\begin{array}{c}\text { DRUG: HPMC } \\
\text { E15 }\end{array}$ & PEG6000 & $\begin{array}{c}\text { SODIUM } \\
\text { CAPRATE }\end{array}$ \\
\hline BNANO1 & $1: 0.25$ & 1.5 & 1.0 \\
\hline BNANO2 & $1: 0.5$ & 1.5 & 1.0 \\
\hline BNANO3 & $1: 0.75$ & 1.5 & 1.0 \\
\hline BNANO4 & $1: 1.0$ & 1.5 & 1.0 \\
\hline BNANO5 & $1: 1.25$ & 1.5 & 1.0 \\
\hline BNANO6 & $1: 1.5$ & 1.5 & 1.0 \\
\hline
\end{tabular}

\section{Standard Calibration Curve of Phenytoin Sodium and Berberine}

Accurately weighed phenytoin $(50 \mathrm{mg})$ was dissolved in $50 \mathrm{ml}$ methanol. Dilutions were made in the range 5,10 , $15,20,25$ and $30 \mu \mathrm{g} / \mathrm{ml}$ and maximum absorbance was monitored at $205 \mathrm{~nm}^{23}$ against the solvent blank ethanol. Similarly, berberine of $50 \mathrm{mg}$ was dissolved in $50 \mathrm{mg}$ of methanol. Dilutions were made with the concentration in the range of $5-30 \mu \mathrm{g} / \mathrm{ml}$ and the absorbance was measured at $345 \mathrm{~nm} .{ }^{24}$ Absorbance values of the Y-axis were plotted against the concentration in $\mathrm{X}$-axis.

\section{FTIR Analysis}

FTIR studies were carried out to study the interaction between formulation components in phenytoinloaded nanoparticles, berberine-loaded nanoparticles and nanoparticles filled capsules FTIR spectrums of phenytoin nanoparticles, berberine nanoparticles and nanoparticles filled capsules were recorded by Shimadzu Fourier Transform Infrared Spectrophotometer (Shimadzu UV 1800). Similarly, KBr disks were formed through compressing powders, at 5 tons of pressure, for $5 \mathrm{~min}$ in the hydraulic press. Most of the readings of spectrum were obtained in between 400 to 4000 per $\mathrm{cm}$.

\section{Differential Scanning Calorimetry (DSC)}

DSC analysis was carried out using model Q-2000 Thermal Analyzer instrument. DSC cell was cleansed by dry nitrogen at $50 \mathrm{ml}$ per min and then the weighed sample (3-5 mg) was placed in an ordinary aluminium pan at 
the temperature of 25 to $300^{\circ} \mathrm{C}$ with the heating rate at $10^{\circ} \mathrm{C} / \mathrm{min}$. Thermograms for phenytoin-loaded and berberine-loaded nanoparticles and nanoparticles filled capsules were recorded based on the melting point of the sample. ${ }^{25}$

\section{Computer Based Drug Interaction Study}

The interaction analyses among the polymer and drug was performed in the biovia discovery studio, 2017 platform. The polymer HPMC E15 (cid: 57503849), berberine (cid: 2353) and phenytoin (cid: 1775) structures were collected from the PubChem database. Initially the grids are generated around the polymer with the coordinates of X (1.152), Y (3.542) and Z (-0.0842). Finally, both drugs are docked around the polymer and the stable complex will be saved for interaction analysis. CHARM based algorithm was used to make an interaction between the drug and polymer.

\section{Molecular Dynamic (MD) Simulation/Interaction of Berberine Nanoparticles}

Molecular dynamic study of Phenytoin nanoparticle andBerberine nanoparticles were evaluated by using BIOVIA Discovery Studio and 2017 and Materials Studio 7.0 software. In this analysis the MD simulations and docking studies were utilized towards investigating the Phenytoin/Berberine-HPMC-PEG nanoparticles formation and the interactions between PEG and also the details of Phenytoin/Berberine drugs in the molecular level.

\section{SEM Analysis}

The diluted phenytoin nanoparticles and berberine nanoparticles were separately examined under Scanning Electron Microscope (Zeiss, Model EVO 18), which was later used to examine the detailed morphological characterization. In this study, the nanoparticles were appended to a carbon tape at the top of SEM aluminium stubs and they were covered with gold by using sputter oater (Electron Microscopy Sciences of model 550X). The Phenytoin nanoparticles and Berberine nanoparticles that were gathered in the extension chamber along with the SEM images of various areas were analysed. The analysis was performed at a high vacuum of $300 \mathrm{~m}$ Torr and the entire SEM experiment was done at an accelerating voltage of $10 \mathrm{kv}$.

\section{Evaluation of Particle Size Andzeta-Potential}

Particle size and zeta-potential of both phenytoin and berberine-loaded nanoparticles were determined by Malvern Zetasizer (By laser light scattering method - Mastersizer-2000; and Malvern, UK). $1 \mathrm{~mL}$ of the diluted nanoparticles were sonicated for $30 \mathrm{sec}$ and placed in the master size cell and later the particle size and zeta-potential were identified.

\section{Determination of Drug Loading and Encapsulation Efficiency}

Prepared phenytoin-loaded or berbering-loaded nanoparticles were centrifuged at $10,000 \mathrm{rpm}$ for $15 \mathrm{~min}$ to isolate the unentrapped drugs. The amount of free drugs in supernatant was quantified spectrophotometrically. Required dilution was done with methanol and the solution was later filtered and the rate of absorbance was measured at 205 and $345 \mathrm{~nm}$, respectively.

\section{In vitro Release Studies}

In vitro release studies were performed in the USP II dissolution test apparatus (Labinda, Disso) with the prepared nanoparticles. In the dissolution chamber, a $900 \mathrm{ml}$ of dissolution media, $0.1 \mathrm{~N}$ HCL of $\mathrm{pH}$ value 1.2 for $2 \mathrm{~h}$ and phosphate buffer of $\mathrm{pH}$ value 6.8 for 24 $\mathrm{h}$ was taken and maintained at $37^{\circ} \mathrm{C} \pm 0.5^{\circ} \mathrm{C}$. Samples were introverted in predetermined intervals upto 24 $\mathrm{h}$ and the equal amount of buffer was replaced. The samples were filtered and the dilution was carried in the concentration of $10 \mathrm{mg} / \mathrm{mL}$ and then the amount of drug released was quantified spectrophotometrically.

\section{Formulation and Evaluation of Capsules Filled with Nanoparticles}

Optimized phenytoin- and berberine-loaded nanoparticles (PNANO-5 and BNANO-5) equivalent to $100 \mathrm{mg}$ were filled in 1" sized hard gelatine capsule.

\section{Weight Variation and Uniformity of Content Test}

20 capsules were weighed with the help of an electronic balance and the experiment was conducted based on the USP official limits. 10 Capsules were weighed and the net contents were removed. Nanoparticles equivalent to 100 mgofphenytoin and berberine were taken in separate $100 \mathrm{~mL}$ volumetric flask, methanol was added and the absorbance was measured at $205 \mathrm{~nm}$ and $345 \mathrm{~nm}$, respectively.

\section{In vitro Release Studies}

In vitro dissolution analyses were carried out for Nanoparticles filled capsules ${ }^{26}$ in dissolution test apparatus (Labinda, Disso) using $900 \mathrm{~mL}$ of dissolution media, $0.1 \mathrm{~N} \mathrm{HCl}$ of $\mathrm{pH}$ value 1.2 for $2 \mathrm{~h}$ and phosphate buffer of $\mathrm{pH}$ value 6.8 for $24 \mathrm{~h}$ at $37 \pm 0.5^{\circ} \mathrm{C}$. Samples were introverted in pre-determined intervals up to $24 \mathrm{~h}$. Equal amount of buffer was replaced. Then the samples were filtered and dilutions were carried on with the concentration of $10 \mathrm{mcg} / \mathrm{ml}$ and the amount of drug released was quantified spectrophotometrically. 


\section{Kinetic Analysis of Drug Release Profiles}

The calculated data from the drug release was fitted into Higuchi and Korsmeyer-Peppas exponential equation, to establish the mechanism of drug release.

$Q=k t^{t}$,

-Where $Q$ is the percentage of drug release, $t$ is time, $k$ is a constant and $n$ is the diffusional exponent which is the significant marker of the mechanism of drug release, from the dosage form.

\section{Acute Toxicity Analysis}

Acute toxicity analysis was conducted for Phenytoin and Berberine nanoparticles to ascertain the safe dose. Six healthy albino rats were selected for the lab test and the selected animals were addressed at Animal House Facility Institute until they attained significant weight of 150-180gm, appropriate for the current investigation. Institutional Animal Ethical Committee approval (IAEC/CHITRAKARTHIKEYINI.S/ AU/1524559770/Ph.D/KMCP/40/2018)was obtained for exploitation of the animals as well as the research design. Phenytoin and Berberine nanoparticles were suspended in DMSO individually and administered (5, 50, 300 and $2000 \mathrm{mg} / \mathrm{kg}$ ) to the Wistar albino rat groups, in a solitary oral dose, through gavages by a feeding needle. The direct group acquired an equal amount of vehicle; observations were noticed systematically and constantly as per OECD guideline-423. The visual examinations such as mobility, skin changes, sensitivity to the pain and sound, aggressiveness and respiratory movements were systematically noticed. Finally, the numbers of survivors (sample rats) were noted down after the first 24h and later the remaining animals were monitored for 14 more days with daily-based observations and reports. The toxicological influence was estimated, on the source of mortality. ${ }^{27}$

Evaluation of Anticonvulsant Activity by MES STUDY Six groups of six Wistar albino rats respectively, with 150-180g of either sex were employed for the current analysis. Group A acquired the vehicle; Group B1, B2 B3, received phenytoin $25 \mathrm{mg} / \mathrm{kg}$, berberine $25 \mathrm{mg} / \mathrm{kg}$ and its free drug combination respectively as a standard; Group C1, C2, C3 received (Test samples) phenytoin-loaded nanoparticles $5 \mathrm{mg} / \mathrm{kg}$, berberine-loaded nanoparticles $5 \mathrm{mg} / \mathrm{kg}$ and phenytoin and berberineloaded nanoparticles together, respectively. Experiment was initiated after an hour of oral treatment by using either the vehicle or the nanoparticles. Equipment with pinna electrodes was employed to deliver the stimuli. Under the strength of stimulus, $45 \mathrm{~mA}, 50 \mathrm{~Hz}$ for $0.2 \mathrm{~s}$, all the rats treated with vehicles revealed the features of extensor tonus. Animals were then monitored intimately for $2 \mathrm{~min}$. It was identified that the vanishing of tonic hind limb extensor was employed, as a positive criterion. Inhibition percentages of seizures related to control was also measured. ${ }^{28}$

\section{Histopathology Study}

\section{Cresylviolet Staining and Morphological Observation of Brain Regions}

Different groups of albino Wistar rats were pre-treated with pure drug and combination, individual nanoparticles and combined nanoparticles (Capsule), respectively. Later on the animals were sacrificed under anaesthesia and the brain-cortex sections were taken for analysis to study the histopathological changes, where the Cresyl violet was used as a histopathologicalstainer. ${ }^{29}$ Cresyl violet stained both neurons and glia and produced a brilliant violet colour as a result. Staining and Morphological observation was carried out in three steps: (i)

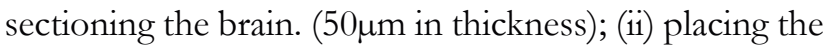
brain sections on the slides and application of the stain and (iii) viewing the stained sections in conjunction with a brain atlas.

\section{RESULTS AND DISCUSSION}

\section{Pre-Formulation Study}

In the pre-formulation study, group of studies has been carried out.

\section{Solubility Study}

Solubility study was carried out with 3 different solubility enhancers such as SLS, Tween-80 and PEG-6000. Among the three, $1.25 \%$ of PEG-6000 increased the phenytoin solubility to 6 -fold and $1.5 \%$ increased the berberine solubility to 7 fold times, respectively. The study established that PEG-6000 was selected as a good candidate for solubility enhancement and it was miscible with all proportions of solvent and doesn't support the microbial growth.

\section{Permeability Study}

The permeability study was carried out with the egg membrane that was clamped in the Franz diffusion cell. Egg membranes assist in optimizing the concentration of the permeability enhancer. Sodium caprate and Berberine mixture were placed in the diffusion cell and then the concentration was optimized. The results thus revealed that a threefold of drug permeation was increased at the concentration level of $1.0 \%$ due to the coupling of sodium caprate with the drug with the appropriate concentration level. 


\section{FTIR Study}

Similarly, the FTIR analysis was utilized to examine the possibilities of the physicochemical interaction between the drug and the polymer. ${ }^{30}$ FTIR spectrums were taken to reveal the interaction of Phenytoin, Berberine and its nanoparticles. In the FTIR spectrum, the peak at $1731.6 \mathrm{~cm}^{-1}$ represents the carbonyl $\mathrm{C}=\mathrm{O}$ stretching of the PEG and the peak at $2912.95 \mathrm{~cm}^{-1}$ and $2848.35 \mathrm{~cm}^{-1}$ represents the methyl and propyl group of HPMC E15. The spectrum of the nanoparticles demonstrated that no characteristic set of peaks interacted; however, it clearly proved that the drugs were encapsulated by HPMC E15 and there were no significant interactions between the drug and the polymer. (Figure 1).

\section{DSC Study}

DSC is utilized to examine any physicochemical interactions between the drug and the polymer. The rapid or drastic changes in shifts of exothermic and endothermic peaks are the sign of interactions between the drug and the polymer. Absence of the drug peak in DSC thermogram indicates the amorphous or solid state of the drug in the polymer. ${ }^{31}$ The Endothermic peak at $196.9^{\circ} \mathrm{C}$ and Exothermic peak at $81.7^{\circ} \mathrm{C}, 115.6^{\circ} \mathrm{C}$ and $186^{\circ} \mathrm{C}$ in Berberine was found to be absent in Berberine nanoparticles; similarly the characteristic set of Exothermic peak at $69.0^{\circ} \mathrm{C}$ and $90.0^{\circ} \mathrm{C}$ of Phenytoin was absent in the Phenytoin nanoparticles. Thus, it was clearly proved that the Phenytoin and Berberine drugs were incorporated in HPMC E15. (Figure 2)

\section{Computer Based Drug Interaction}

The computer-based interaction studies between the polymer and the drug projected the molecular levelinsight view and the nature of the drug's stability. Results of the interaction showed that, both the drugs (Berberine and Phenytoin) interacted well with the poly-

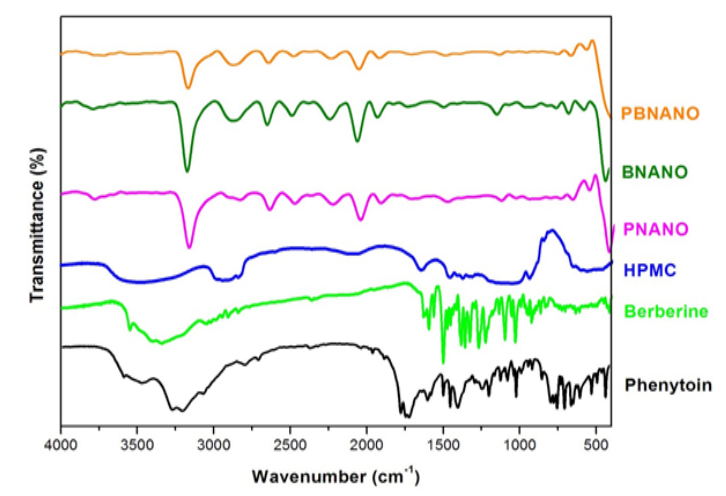

Figure 1: FTIR Spectra of: a) Phenytoin b) Berberine c) HPMC E15 d) Phenytoin nanoparticles e) Berberine nanoparticles f) Capsule filled Phenytoin and Berberine nanoparticles. mer. Analysis of the HPMC E15 polymer and Berberine revealed that, three interactions are formed in the complex, they are: two-hydrogen in the Dioxa ring of the Berberine formed a hydrogen bond with the Oxygen atoms of HPMC; one hydrophobic $\pi$ interaction was generated between benzene ring of berberine and hydrogen atom of polymer; Similarly, the Phenytoin formed three hydrogen bond interaction with the range of $2.06-2.60 \AA$ distance and one hydrophobic $\pi$ interaction with the polymer. Thus, the results clearly proved that both the drugs formed stable complex with HPMC E15polymer (Figure 3 and 4)

\section{Simulation/interaction of Phenytoin and Berberine Nanoparticles}

In this work, the MD simulations and docking studies were used to investigation the Phenytoin/ Berberine- HPMC E15-PEG nanoparticle formation and the interactions between PEG and the Phenytoin/ Berberine drugs in molecular level details. The first aim of this study was the validation of this new MD simulations procedure, which was tested on two drugpolymer systems: (a) Phenytoin - HPMC E15-PEG (b) Berberine- HPMC E15-PEG. Before MD simulations, the Force field (COMPASS) was analysed for Berberine, Phenytoin, HPMC and PEG. It shows the Forcite energy of $4092113472.7683 \mathrm{Kcal} / \mathrm{mol}$ (Berberine), $232326104289041750 \mathrm{Kcal} / \mathrm{mol}$ (Phenytoin), $131430524.73022 \mathrm{Kcal} / \mathrm{mol}$ (HPMC) and 7953.42717 $\mathrm{Kcal} / \mathrm{mol}$ (PEG). Berberine-HPMC E15- PEG and Phenytoin- HPMC E15-PEG complex molecules are built in an Amorphous cell protocol to simulate the interaction within the Berberine and Phenytoin nanoparticle. (Figure 5).

The Berberine molecule forms van der Waals energy of 565.235 with HPMC and PEG molecules. The aromatic rings in the Berberine molecules form highest van

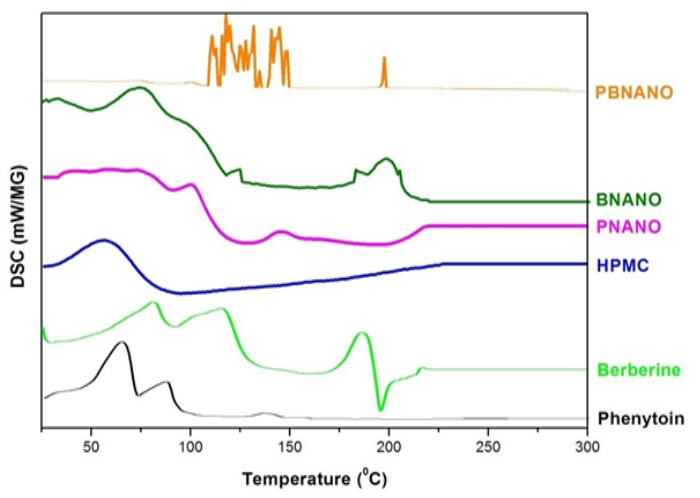

Figure 2: DSC Spectra of: a) Phenytoin b) Berberine c) HPMC E15d) Phenytoin nanoparticles e) Berberine nanoparticles f) Capsule filled Phenytoin and Berberine Nanoparticles. 

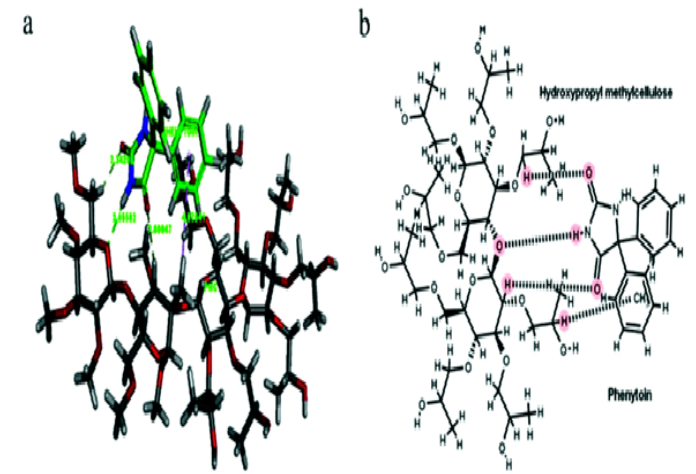

Figure 3: 3D and 2D Interaction Pattern of the HPMC E15 Polymer and Phenytoin.
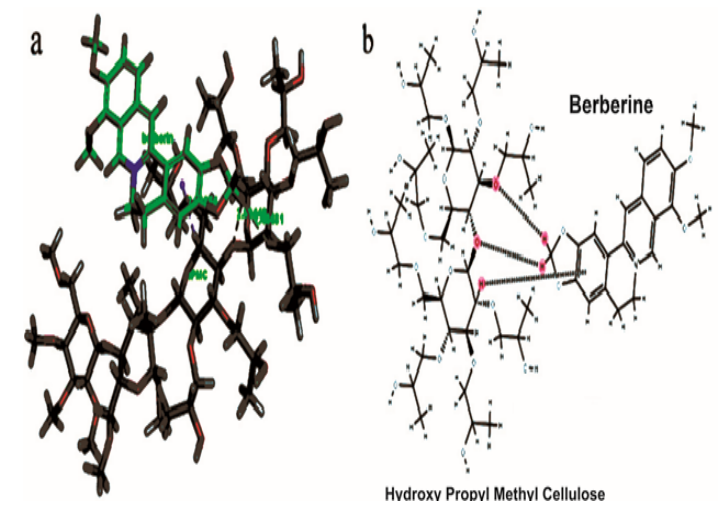

Figure 4: 3D and 2D Interaction Pattern of the HPMC E15 Polymer and Berberine.

der Waals of energy with hydrophobic groups of the HPMC E15 and PEG molecules. Similarly, the two aromatic rings of the Phenytoin molecule forms van der Waals of the energy of 716.991 with HPMC E15and PEG molecules. During the molecular interaction analysis, the H-bond of the both HPMC E15 and PEG molecules were fixed as a stable condition to improve the Berberine and Phenytoin interactions. Additionally, the involved van der Waals interactions between the drug and polymers are accountable for nanoparticle formations. (Figure 6).

The Standard Dynamics Cascade Protocol in BIOVIA Discovery Studio shows the time-based temperature and total energy values for Berberine and Phenytoin nanoparticle compositions. From the result of molecular dynamics, the Berberine with HPMC E15 and PEG found the energy of the nanoparticle composition at the stabilized state of $136.568 \mathrm{Kcal} \mathrm{mol}^{-1}$ and the temperature stabilized at $295 \mathrm{~K}$. Similarly, Phenytoin stabilized at energy of $100 \mathrm{Kcal} \mathrm{mol}^{-1}$ and temperature stabilized at $320 \mathrm{~K}$. This results, revealed that the stability of the

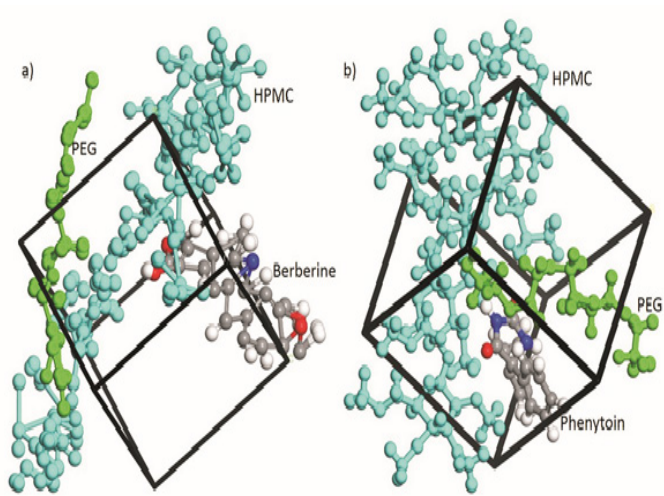

Figure 5: Formation of Molecular Interactions Between (a) Berberine nanoparticle and (b) Phenytoin nanoparticles simulated by Amorphous cell modules construction and Packing method using Material Studio Software.

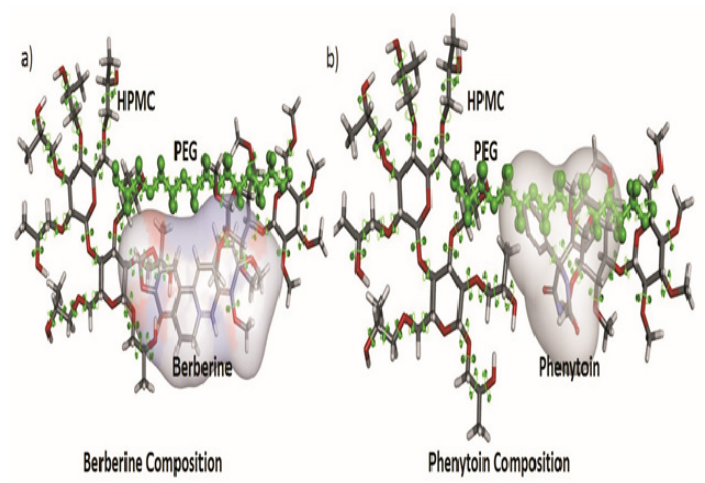

Figure 6: Van der Waals Interactions between (a) Berberine Composition and (b) Phenytoin Composition.

both Berberine and Phenytoin nanoparticles which remains unaffected by the physical condition. (Figure 7).

\section{SEM Study}

Surface Morphology was identified through the SEM photograph-study. It showed that the particles of Phenytoin and Berberine were nano-sized and they were in uniformly spherical in shape (Figure 8 and 9).

\section{Particle Size with Zeta-Potential}

Distribution of particle size by Zeta sizer proved that, all the particles were found to be smooth and uniformly spherical in shape; also it exists within the range of 100 - 500nm.Zeta-potential of Phenytoin and Berberine nanoparticles were $-2.61 \mathrm{mv}$ and $-35.9 \mathrm{mv}$. The negative side of zeta-potential indicated that the system is stable. Poly Dispersity Index (PDI) of all the nanoparticles existed within the range of 1 indicated that there is no aggregation of system. (Figure 10 and 11) (Table 3).

\section{Entrapment Efficiency and Drug Loading}

In this analysis the drug was entrapped in HPMC E15, depending upon the solubility and polarity proper- 
Table 3: In vitro Release, Drug Loading and Encapsulation Efficiency, Particle size and Poly Disperse Index for All Batches.

\begin{tabular}{|c|c|c|c|c|c|}
\hline Formulation code & $\begin{array}{c}\text { In vitro release } \\
\text { at } 24^{\text {th }} \mathrm{hr}\end{array}$ & $\begin{array}{c}\text { Drug } \\
\text { Loading \% }\end{array}$ & $\%$ EE & Particle size & Polydispersity index \\
\hline PNANO1 & $84.2 \%$ & 20.0 & 60.1 & $1025 \mathrm{~nm}$ & 0.702 \\
\hline PNANO2 & $90.0 \%$ & 22.7 & 68.2 & $865 \mathrm{~nm}$ & 0.540 \\
\hline PNANO3 & $93.5 \%$ & 25.13 & 75.4 & $745 \mathrm{~nm}$ & 0.050 \\
\hline PNANO4 & $96.4 \%$ & 26.3 & 79.1 & $520 \mathrm{~nm}$ & 0.205 \\
\hline PNANO5 & $98.0 \%$ & 29.46 & 88.4 & $321 \mathrm{~nm}$ & 1.000 \\
\hline PNANO6 & $97.9 \%$ & 28.8 & 86.4 & $640 \mathrm{~nm}$ & 0.547 \\
\hline BNANO1 & $86.2 \%$ & 17.8 & 62.3 & $985 \mathrm{~nm}$ & 0.080 \\
\hline BNANO2 & $93.5 \%$ & 20.02 & 70.1 & $752 \mathrm{~nm}$ & 0.476 \\
\hline BNANO3 & $95.6 \%$ & 21.48 & 75.2 & $600 \mathrm{~nm}$ & 0.520 \\
\hline BNANO4 & $97.5 \%$ & 23.51 & 82.3 & $541 \mathrm{~nm}$ & 0.471 \\
\hline BNANO5 & $98.5 \%$ & 25.74 & 90.1 & $471 \mathrm{~nm}$ & 0.592 \\
\hline BNANO6 & $98.1 \%$ & 25.25 & 88.4 & $510 \mathrm{~nm}$ & 0.740 \\
\hline
\end{tabular}

Results are depicted as mean $\pm \mathrm{SD}(n=3)$.
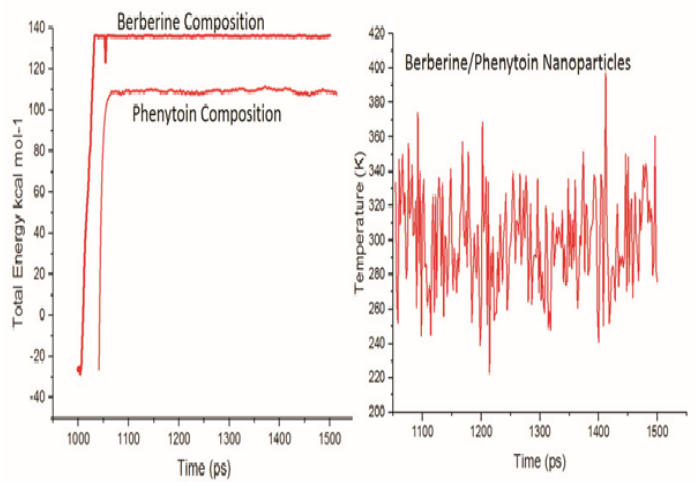

Figure 7: Time-Based Total Energy and Temperature Changes Graph in Molecular Dynamics Study.

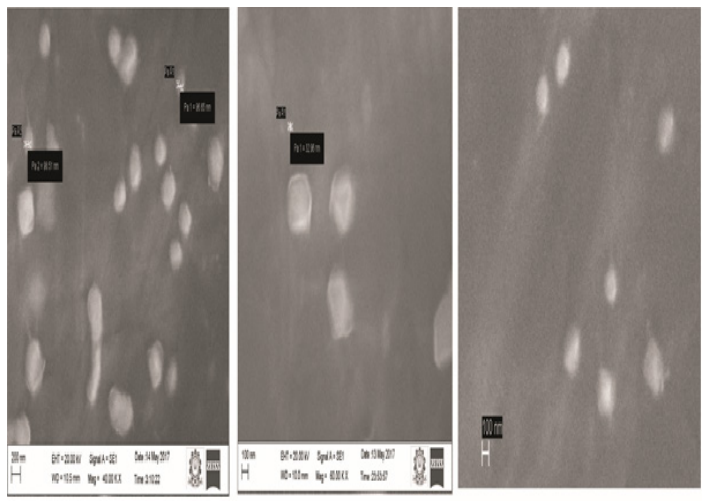

Figure 8: SEM image of Phenytoin Nanoparticles.

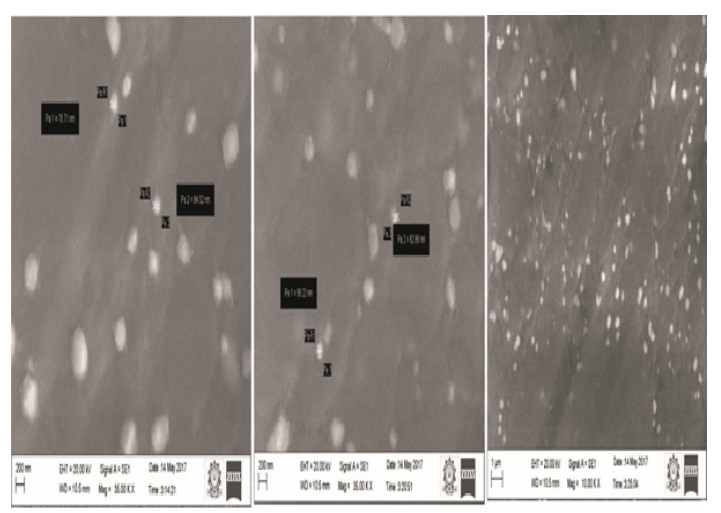

Figure 9: SEM Image of Berberine Nanoparticles.

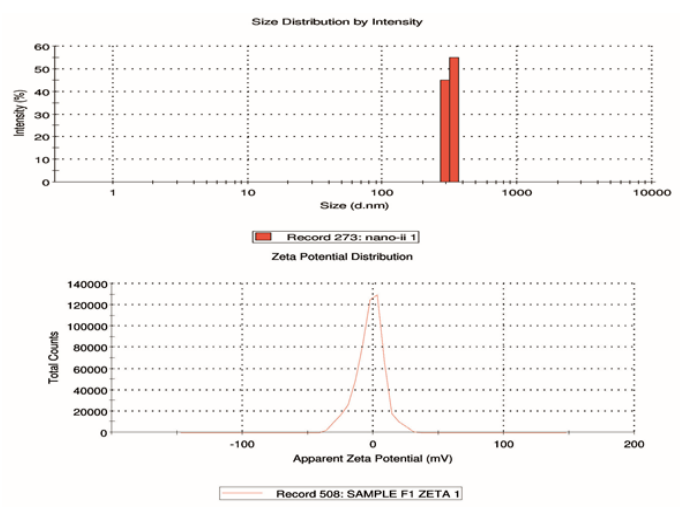

Figure 10: Particle Size and Zeta Potential of Phenytoin Nanoparticles. 
ties. The gathered data shows that: if the particle size increases, the entrapment efficiency decreases, respectively; similarly, the drug loading and entrapment efficiency for PNANO-5 was estimated as $29.4 \%$ and $88.4 \%$ and BNANO-5 was estimated as $25.74 \%$ and $90.1 \%$, respectively. Thus, the results showed a good electrostatic interaction between the drug and the polymer. Particulars of the Drug Loading and Entrapment Efficiency of all the tested batches have been tabulated (Table 3) for further references.

\section{In vitro Dissolution Study}

The dissolution analysis was performed with $0.1 \mathrm{~N}$ HCL of $\mathrm{pH}$ value of 1.2 for $2 \mathrm{~h}$ and phosphate buffer of $\mathrm{pH}$ value 6.8 for $24 \mathrm{~h}$. Dissolution profile showed that PNANO-5 and BNANO-5 were the optimized formulation and the percentage drug release of PNANO-5 was $98 \%$ whereas the BNANO-5 was $98.5 \%$.Increased concentration of HPMC E15 releases the drug, slowly and extended the release up-to 24h. [Table 3].

\section{Evaluation of Capsules}

Various tests and studies were done and the capsules were evaluated under certain norms.

Weight Variation Test and Uniformity of Content

All the capsules were within the limit of official standard as per I.P.

\section{In vitro Dissolution of the Prepared Capsules}

The dissolution profile of Phenytoin and Berberine nanoparticles showed $98.0 \%$ and $98.5 \%$ drug release respectively and later the release was extended up-to 24 $h$. Thus, the release data showed that the percentage of the drug release was increased for nanoparticles when it is compared to that of pure drug. The obtained result demonstrated that the release was modified and controlled and it could be attributed by the size of nanoparticles and in the increase of surface area between the solvent and the drug surface.

\section{Kinetics of Drug Release}

To determine the drug release, different kinetic models (Such as, zero order, first order, etc) were adapted from the study of Higuchi and also the Korsmeyer-Peppas equations were obtained too. The percent release was plotted against time to get zero order plots similarly the log percent remaining was plotted against time to get first order plot; additionally, the percent release was plotted against square-root of time to get the Higuchi plot. Finally, the log cumulative $\%$ drug release was plotted against log time in hours, towards attaining the Korsmeyer-Peppas plot.
Optimized Formulations (PNANO-5 and BNANO5) has highest regression coefficients which are 0.975 and 0.976, towards Higuchi's model indicating Fickian diffusion. The diffusion mechanism was established by fitting the data into Korsmeyer-Peppas equation and thus the regression coefficients of Korsmeyer-Peppas plot of optimized formulations are estimated at 0.892 and 0.898 . The analysis showed good linearity and the diffusion was identified as the predominant mechanism of the drug release. The values of the release exponent (n) of PNANO-5 and BNANO-5 formulations are of 0.351 and 0.354 , respectively and the results indicated that $n$-value is around 0.5 . Henceforth, it was proved that the drug release follows Fickian diffusion (Figure 12).

\section{Pharmacological Evaluation}

\section{Acute Toxicity}

Once the drugs were successfully administrated in the selected animals, they were monitored and observed for 14 consecutive days and all examinations were recorded systematically and individual records were preserved for each animal, respectively. Variations of fur, skin, respiratory circulatory, eyes and mucous membranes, central and autonomic nervous systems, behaviour pattern and somato-motor activity were monitored and recorded. In this acute toxicity analysis, Phenytoin and Berberine nanoparticles were established to be toxic at $1000 \mathrm{mg} /$ $\mathrm{kg}$ and whereas to be secure up to $5 \mathrm{mg} / \mathrm{kg}$, in wistar albino rats.

\section{MES study}

The Tonic hind limb extension (THLE) occurrence was a positive parameter for MES study. THLE abolition was obtained as a safeguard technique against the MES seizures and THLE incidence along with the time of THLE in seconds were also observed for better understanding. The Statistical Analysis was conducted through "Graph Pad Prism 5" technique (San Diego,

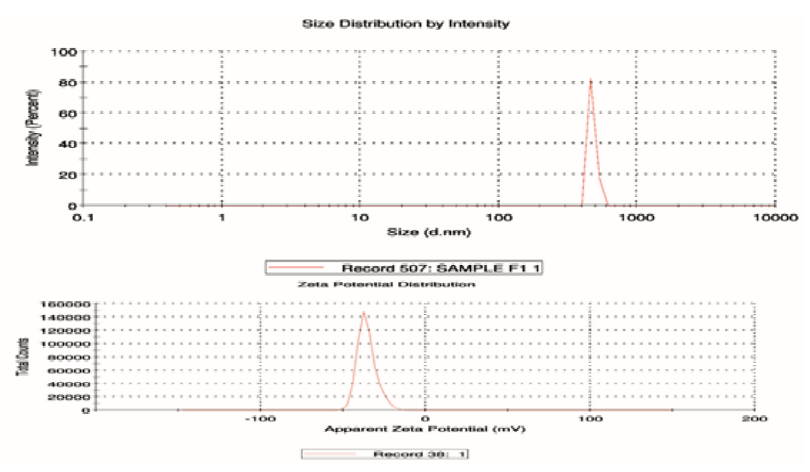

Figure 11: Particle size and Zeta Potential of Berberine Nanoparticles. 
California, USA). On other-hand One-Way ANOVA was employed with post-hoc Turkey's experiment, for comparison among the groups. The $P<0.05$ was assumed as important.

THLE arrival of action and time of action along with the test drugs such as Phenytoin nanoparticles, Berberine nanoparticles, combination of free drug and Capsules (Phenytoin and berberine combination) were recorded for standardization. The results showed that, individual nanoparticles have significant efficacy than pure drug by reducing THLE duration $(P<0.001)$. Free Phenytoin and Berberine showed slightly higher efficacy than individual nanoparticles; but Phenytoin and Berberine nanoparticles Combination was found to be more efficacious than free drugs combination and inhibit the convulsion remarkable level. Thus, the results and reports proved that efficacy has been improved with the prepared capsules. The arrival and time of THLE (Tonic Hind Limb Extension) and inhibition percentage of convulsions were noted. [Table 4] (Figure 13).

Where Mean value $\pm \mathrm{SD}(n=6)$. Statistical significance was determined by ANOVA followed by Dunnett'stest, where $($ THLE $=$ Tonic hind limb extension) $* P<0.05$.

\section{Histopathological Study}

In Control cortex regions, the test rats showed decrease in neuronal cells and increase in chromatolysis and pyknotic nuclei. It has been observed through the analysis that Phenytoin $25 \mathrm{mg} / \mathrm{kg}$ (i.p.) and Berberine 25mg/ $\mathrm{kg}$ (i.p.) significantly decreased the neuronal cell death, brain cell oedema, chromatolysis and pyknotic nuclei in each and every region when compared against the control rats' cortex regions. Similarly, the rats that are treated with individual nanoparticles showed significant decrease in cell edema and neuronal cell degeneration in each and every region when compared to the control rats. Thus, the rats treated with the combination of Phenytoin and Berberine nanoparticles showed significant decrease in neuronal cell death with mild edematous nuclei in all regions as compared with combination of free drugs. (Figure 14).
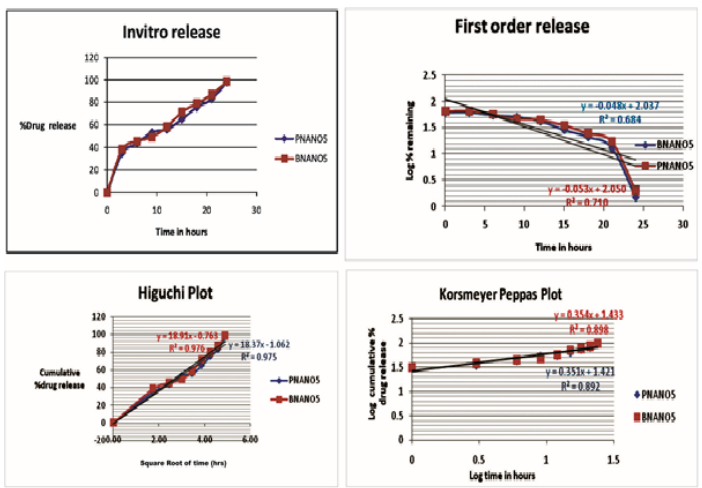

Figure 12: Kinetics of Release.

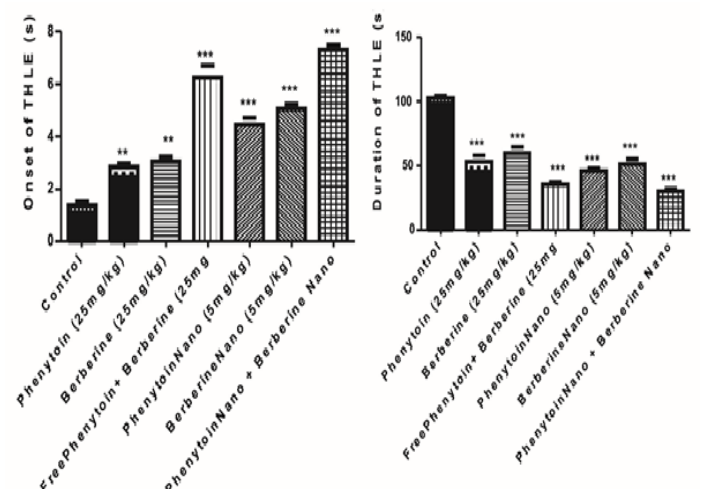

Figure 13: Onset and Duration of Convulsion Estimation by MES Study.
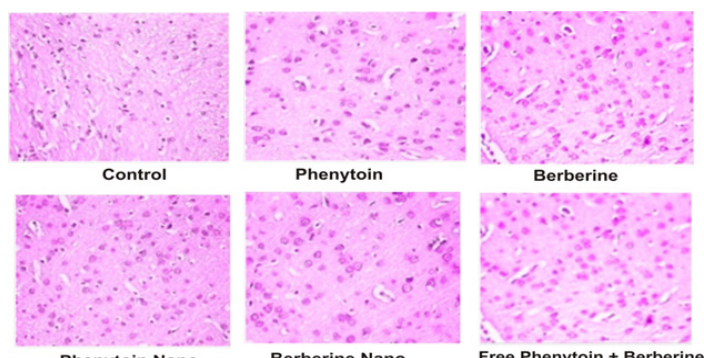

Phenytoin Nano

Berberine Nano

Free Phenytoin + Berberin

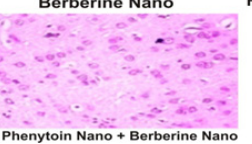

Figure 14: Histopathological Images for Pure Drug, Plain Nano Particles and Combinations of Free Drug and Nanoparticles.

\begin{tabular}{|c|c|c|c|}
\hline \multicolumn{3}{|c|}{ Table 4: Effect of Phenytoin, Berberine Pure Drug, Plain Nanoparticles and Combinations of Free Drug and } \\
Nanoparticles on MES Induced Convulsion in Rats.
\end{tabular}




\section{CONCLUSION}

In this research great effort has been taken to improve the efficacy of Phenytoin anticonvulsant activity. In order to accomplish the goal, controlled release individual Phenytoin and Berberine nanoparticles were prepared and filled in with capsules to make them a single-unit dosage form. MES study thus proved that combination therapy in capsule is significantly efficacious than Phenytoin mono-therapy and its $24 \mathrm{~h}$ drug release design thus makes simple administration and reduces the dosage frequency. The research thus argues and proves that the developed capsules are more beneficial in Status Epilepsy than individual Phenytoin therapy.

\section{ACKNOWLEDGEMENT}

The study was conducted at Anna University, Thiruchirappalli and part of work at KM College of Pharmacy, Madurai and the authors wish to offer their sincere gratitude to Mrs. Ruckmani Kandasamy and Mr. Subramanian Natesan, Anna University, Thiruchirappalli and Mr. Nagarajan and Hariharan, K.M. College of Pharmacy, Madurai, Mr. Saisivam, NRV enkaria institute of Pharmacy, Junagadh, Gujarat, for providing sufficient laboratory facilities and valuable guidance's to carry out the experiment.

\section{CONFLICT OF INTEREST}

The authors declare that they have no conflict of interest.

\section{ABBREVIATIONS}

THLE: Tonic Hind Limb Extension; MES study: Maximum Electrical Shock study; P-gp: P-glycoprotein; CNS: Central Nervous system; PEG: Poly Ethylene Glycol; CYP: Cytochrome P-450.

\section{REFERENCES}

1. Chang RC, Hudson PM, Wilson BC, Liu B, Abel H, Hong JS, et al. High concentrations of extracellular potassium enhance bacterial endotoxin lipopolysaccharide-induced neurotoxicity in glial neuron mixed cultures. Neuroscience. 2000;97(4):757-64. PMID: 10842021 [PubMed]

2. Patel M. Mitochondrial dysfunction and oxidative stress: cause and consequence of epileptic seizures. Free Radic Bio Med. 2004;37(12):195162.

3. Matsuki N, Quandt FN, Ten ERE, Yeh JZ. Characterization of the block of sodium channels by phenytoin in mouse neuroblastoma cells. Journal of Pharmacol Exp Thera. 1984;228(1984):523-40. http//www.nebi.nlm.nih.gov/ pubmed/63196812.

4. World Health Organization, Epilepsy in the WHO Africa Region, Bridging the Gap: The Global Campaign against Epilepsy: "Out of the Shadows", Geneva, Switzerland. 2004.

5. Karunakar H, Thakker SP, Joshi AB, Shastry CS, Chandrasekhar KS. Anticonvulsant activity of Carissacar and as Linn, Root Extract in Experimental Mice. Trop J Pharm Res. 2009;8(2):117-25. http://dx.doi. org/10.4314/tjpr.v8i2.44519
6. Robertt MK. Nelson text book of Pediatrics, evaluationof 1" seizure and definition of seizure19th edition, Elsevier Inc. 1978;586.

7. McNamara $O$. Drugs effective in the therapy of the epilepsies. The Pharmacological Basis of Therapeutics. McGraw Hill, New York. 1996;47885.

8. Caroline F, Michelle W, Steven L, Pharma G. summary: phenytoin pathway. Pharmacogenetics and Genomics. 2012.

9. Sills GJ. Antiepileptic Drug Transport- of Mice and Men. Epilepsy Curr. 2008;8(2):48-50.doi:10.1111/j.1535-7511.2008.00234.x

10. Robert D, Hrvoje J, Gordana B. Hepatoprotective activity of berberine is mediated by inhibition of TNF- COX-2 and iNOS expression in $\mathrm{CCl}_{4}$ intoxicated mice. Toxicology. 2011;280(1-2):33-43.1https://doi.org/0.1016/j. tox.2010.11.005

11. Hua-Wen X, Xia T, Meng O, Jian X, Wei-Lang L. Effects of berberine on pharmacokinetics of midazolam and rhodamine 123 in rats in vivo. Springer Plus. 2016;5(1):380. https://doi.org/10.1186/s40064-016-2013-Z

12. Zhao $X$, Zhang JJ, Wang $X$, Bu XY, Lou YQ, Zhang GL. Effect of berberine on hepatocyte proliferation, inducible nitric oxide synthase expression, cytochrome P450 2E1 and 1A2 activities in diethylnitrosamine- and phenobarbital-treated rats. Pharmacol Ther. 2008;62(9):567-72. https://doi. org/10.1016/j.biopha.2007.02.009

13. Chang $\mathrm{Z}$, et al. Inhibition of CYP450 $1 \mathrm{~A}$ and $3 \mathrm{~A}$ by berberine in crucian carp Carassiusauratusgibelio. Comparative Biochemistry and Physiology Part C. 2011;154(4):360-6. https://doi.org/10.1016/j.cbpc.2011.07.005

14. Tourandokht BM, Roghani M. The Anticonvulsant and Antioxidant Effects of Berberine in Kainate-induced Temporal Lobe Epilepsy in Rats. Spring. 2014;5(2):124-30. PMID: 25337370

15. Wang HC, Wang BD, Chen MS, Chen H, Sun CF, Shen G, et al. Neuroprotective effect of berberine against learning and memory deficits in diffuse axonal injury. Experimental and Therapeutic Medicine. 2018;15(1):1129-35. https:// doi.org/10.3892/etm.2017.5496

16. Kreuter J. Drug delivery to the central nervous system by polymeric nanoparticles: What do we know?. Advanced Drug Delivery Reviews. 2014;71:2-14. https://doi.org/10.1016/j.addr.2013.08.008

17. Papich MG, Martinez MN. Applying Biopharmaceutical Classification System (BCS) Criteria to Predict Oral Absorption of Drugs in Dogs: Challenges and Pitfalls. The AAPS Journal. 2015;17(4):984-64. https://doi.org/10.1208/ s12248-015-9743-7

18. Modi A, Ttayade P. A Comparative solubility enhancement profile of valdecoxib with different solubilisation approaches. Indian Journal of Pharmaceutical Sciences. 2007;69(2):274-78. https://doi.org/10.4103/0250-474X.33156.

19. Mesnukul AK, Yodkhum K, Phaechamud T. Solid Dispersion Matrix Tablet Comprising Indomethacin-PEG-HPMC Fabricated with Fusion and Mold Technique. Indian Journal of Pharmaceutical Sciences. 2009;71(4):413-20. https://doi.org/10.4103/0250-474X.57290

20. Meng Z, et al. Amorphous solid dispersion of berberine with absorption enhancer demonstrates a remarkable hypoglycemic effect via improving its bioavailability. International Journal of Pharmaceutics. 2014;467(1-2):50-9.

21. Sanjeev R, Elizabatho J, Irene M, Thirumala G. Comparative buccal permeability enhancement of didenosine and tenofovir by potential multifunctional polymeric excipients and their effects on porcine buccal histology. Pharmaceutical Development and Technology. 2014;19(1):82-90. https://doi.org/0.3109/10837450.2012.752505

22. Stephanie D, et al. The Design of Nanoparticles Obtained by Solvent Evaporation: A Comprehensive study. Langmuir. 2003;19(22):9504-10. https://doi.org/10.1021/la034999q

23. Mantri MA, Shanmukhappa S. UV spectrophotometric estimation of phenytoin sodium in pure and pharmaceutical formulations. Asian Journal of Chemistry. 2010;22(3):2447-9.

24. Girish S, Seth AK, Ghanshyam P, Sachin S, Ankur J. Formulation and in vitro evaluation of berberine containing liposome optimized by 32 full factorial designs. Journal of Applied Pharmaceutical Science. 2015;5(07):023-8. https://doi.org/10.7324/JAPS.2015.50704

25. Averineni RK, Shavi GV, Gurram AK, Deshpande PB, Arumugam K, Maliyakkal M. PLGA 50:50 nanoparticles of paclitaxel: development, in vitro anti-tumor activity in BT-549 cells and in vivo evaluation. Bull Mat Sci. 2012;35(3):319-26. 
Ahmed TA. Preparation of finasteride capsules-loaded drug nanoparticles: formulation, optimization, in vitro and pharmacokinetic evaluation. Int $\mathrm{J}$ Nanomedicine. 2011;11:515-27.

27. OECD (Organization for Economic Cooperation and Development), Health Effects: Test No. 423: Acute Oral toxicity - Acute Toxic Class Method. OECD Section 4. 2002. http://dx.doi.org/10.1787/9789264071001-en

28. Mittal R. Antiepileptics. Drug Screening Methods. $2^{\text {nd }}$ ed. New Delhi: Jaypee Brothers Medical Publishers. 2009;400-22.

29. Naida P, Matthew B, Sofoklis P, Revers D, Martine H. A Rapid Method Combining Golgi and Nissl Staining to Study Neuronal Morphology and
Cytoarchitecture. Journal of Histochem Cytochem. 2008;56(6):539-50. https://doi.org/10.1369/jhc.2008.950246

30. Sahoo S, Chakraborti CK, Behera PK, Mishra SC, Raman FTIR Spectroscopic investigations of a Norfloxazacin/ Carbopol934 polymeric suspension. Journal of Young Pharmacists. 2012:(4):138-45. https://doi. org/10.4103/0975-1483.100017

31. Shah B, Kakumanu V, Bansal AK. Analytical techniques for quantification of amorphous/crystalline phases in pharmaceutical solids. Journal of Pharmaceutical Sciences. 2006;95(8):1641-65. [PubMed] https://doi. org/10.1002/jps.20644

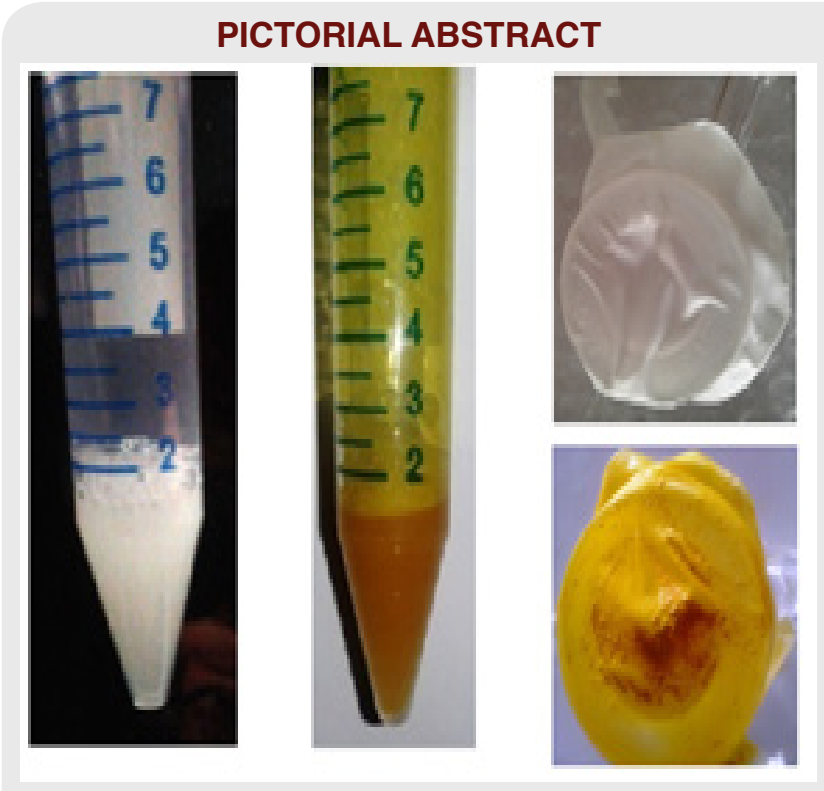

\section{ABOUT AUTHORS}

- Most of anticonvulsant drugs are not explore its total efficacy. Phenytoin is a classic example and its efficacy is inhibited by high metabolization (90\%-95\%) and back transportation through cytochrome P450 and P-glycoprotein, respectively. So new approach has been carried out to improve the efficacy of phenytoin by adopting nano technology and combination therapy. Berberineis a co-drug and has a anticonvulsant activity. It also act as a Cox-2 inhibitor, cytochrome P-450 inhibitor which helps to maximize the free drug(phenytoin) concentration in plasma by reducing metabolization and back transportation. On account of this Phenytoin and berberine nanoparticles were prepared individually and filled in hard gelatine capsule to make as a single unit dosage form. Characterization study revealed that formulations were in nano level Pharmacological investigation such as MES and Histopathological study clearly proved that combination therapy in capsule has improved efficacy than single phenytoin therapy. Hence it is more beneficial in status epileptics than individual phenytoin.

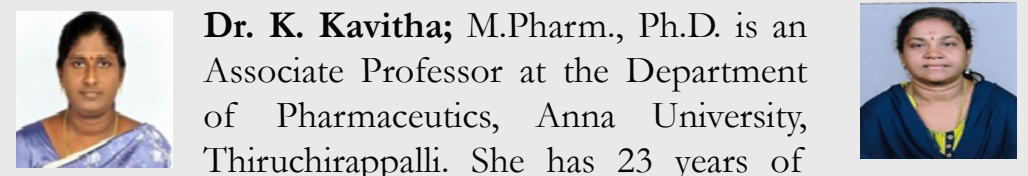
Thiruchirappalli. She has 23 years of teaching experience and Gold Medalist in M. Pharm and Received Best Outgoing Student Award in M. Pharm. She has published many research papers and organized national and international conferences.
Chitra Karthikeyini. S is a Research scholar at the Department of Pharmaceutical Technology, Anna University, Thiruchirappalli.

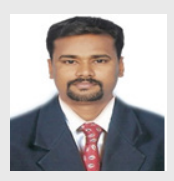

J. Muthu Mohamed is a Research Scholar at the Department of Pharmaceutical Technology, Anna University, Thiruchirappalli.

Cite this article: Senthilvel CK, Karuppaiyan K, Moideen MMJ. Development of Capsules Filled with Phenytoin and Berberine Loaded Nanoparticles- a New Approach to Improve Anticonvulsant Efficacy. Indian J of Pharmaceutical Education and Research. 2019;53(3):468-79. 